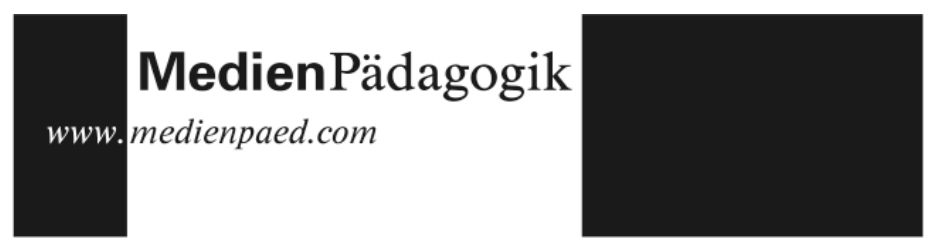

\title{
Rezensionen
}

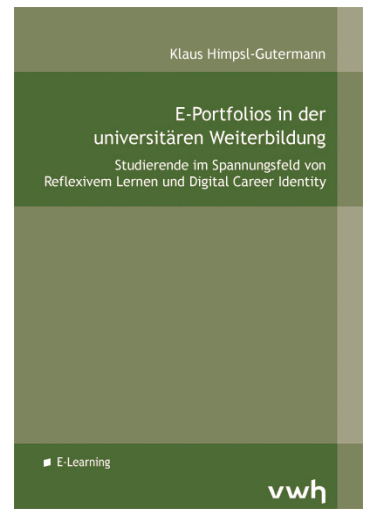

Klaus Himpsl-Gutermann

E-Portfolios in der universitären Weiterbildung. Studierende im Spannungsfeld von Reflexivem Lernen und Digital Career Identity

Zugleich: Diss., Universität Klagenfurt 2012

Boizenburg: vwh, 2012. 313 Seiten

ISBN: $978-3-86488-014-8$

$€$ 30.90; CHF 37,90

\section{Lernprozesse mit E-Portfolios begleiten: Herausforderungen bei der Implementierung im Kontext Hochschule}

\section{Einblicke in den fachwissenschaftlichen Diskurs}

Das Portfolio ist eine Methode zur Sammlung, Darstellung und Bewertung von Lerninhalten, die in formalen Bildungskontexten vielfach als «Portfoliomappe» zum Einsatz kommt und sowohl zu Prüfungszwecken als auch zur Dokumentation und Reflexion von Lernprozessen genutzt wird. Die Portfolioarbeit geht auf reformpädagogische Grundideen zurück, die auch in der digitalen Form beibehalten und um Möglichkeiten der öffentlichen Sichtbarkeit sowie um (Peer-)Feedback ergänzt werden. Inzwischen existieren viele gute Beispiele für den Einsatz von E-Portfolios, allen voran im Hochschulbereich, wo sich die E-Portfolio-Arbeit als günstig erwiesen hat, um primär am Wissensbestand orientierte Prüfungsformen um kompetenzorientierte Verfahren zu erweitern. E-Portfolios werden dort vielfach als Werkzeug zur Bewältigung lehr-lernbezogener Herausforderungen genutzt; in manchen Konzepten ist auch zu erkennen, dass E-Portfolios neue Bildungsräume durch die Repräsentation von Inhalten im Internet und durch den Diskurs mit Lehrenden sowie mit Peers erschließen sollen (z.B. Egloffstein, Baierlein \& Frötschl, 2010; Meyer, Mayrberger, Münte-Goussar \& Schwalbe, 2011). Je nach Intention und Kontext werden unterschiedliche Fragen zur Bedeutung des E-Portfolio-Einsatzes aufgeworfen, die in der Regel bei konkreten Einsatzszenarien in der Praxis anknüpfen und somit den pädagogischen Kern der Portfolioarbeit aufgreifen. 


\section{Inhaltlicher Überblick über die Dissertationsschrift}

In die oben angerissene Diskussion um E-Portfolios fügt sich die Dissertationsschrift zu E-Portfolios in der universitären Weiterbildung von Klaus Himpsl-Gutermann nahtlos ein, die im Jahr 2012 im Verlag Werner Hülsbusch erschienen ist. Die Arbeit unternimmt den Versuch, «das Potenzial, das dem elektronischen Portfolio als Begleiter des lebenslangen Lernens zugeschrieben wird» (Himpsl-Gutermann, 2011, 17) auszuloten. Die Grundannahme spiegelt sich in der Zielsetzung der Arbeit und in den Forschungsfragen, die auf den weiterbildenden Masterstudiengang eEducation an der Donau-Universität Krems (Österreich) fokussieren und konkret (1) nach Gestaltungsmöglichkeiten von E-Portfolios suchen, (2) die Einbettung von EPortfolios unter technischen und didaktischen Gesichtspunkten erfassen sowie (3) die Implementierung am Fall untersuchen. Das Erkenntnisinteresse lässt sich grob in zwei Richtungen zusammenfassen: einerseits verortet sich die Arbeit in der Weiterentwicklung und Reflexion des Studiengangs eEducation, andererseits will sie einen empirischen Beitrag zur E-PortfolioForschung liefern (ebd., 25). Um die Forschungsfragen zu beantworten, nutzt der Autor ein qualitatives Forschungsdesign, das auf einem Aktionsforschungsprojekt basiert und Anleihen bei der Grounded Theory nimmt. Dieser qualitative Zugang wird bereits im Aufbau der Arbeit sichtbar: Nach der Vorstellung des theoretischen wie auch praktischen Kontexts wird der Kremser E-Portfolio-Ansatz näher skizziert (Kapitel 1 bis 5). Im Anschluss daran gewinnt die empirische Untersuchung des Einzelfalls an Bedeutung, die die Vorarbeiten des Autors etwa hinsichtlich einer Taxonomie von E-Portfolios sowie der Evaluation von E-Portfolio-Software ergänzen (Kapitel 6 bis 8). Die Arbeit endet mit Forschungsdesideraten sowie einer Zusammenfassung der Ergebnisse (Kapitel 9 und 10).

Ausgehend von sieben qualitativen Interviews sowie (Dokumenten)Analysen von E-Portfolios entwickelt Himpsl-Gutermann ein 4-PhasenModell der E-Portfolio-Nutzung. Darin lassen sich die Phasen «sich orientieren», «sich positionieren», «sich identifizieren» und «sich präsentieren» 
deduktiv voneinander unterscheiden. In allen Phasen werden nach innen und nach außen gerichtete Nutzungsmotive der Person bei der E-PortfolioNutzung identifiziert, die den Status des E-Portfolios als Schnittstelle zwischen Reflexionswerkzeug und medialem Bildungsraum, etwa durch PeerFeedback oder die Eröffnung neuer Perspektiven auf das eigene Lernen, verdeutlichen (ebd., 133). Durch die Verknüpfung der genannten Nutzungsmotive kommt der Verfasser zu dem Schluss, dass sich die E-PortfolioArbeit innerhalb des von inm untersuchten Falls zwischen «Digital Identity" und "Career-Identity» bewegt (ebd., 130). Er deutet allerdings auch Nutzungsunterschiede bei den involvierten Studierenden an, die mit den Funktionen der E-Portfolio-Arbeit (von Heuristik über Organisation bis hin zu Wissenstransfer etc.) einhergehen. Die technischen Voraussetzungen von EPortfolio-Software belaufen sich auf eine 3-Schichten-Struktur: So sollte diese die Möglichkeit bieten, Artefakte abzubilden, Reflexion durch Journaleinträge zu erleichtern und ausgewählte Lernergebnisse auch nach außen zu repräsentieren (ebd., 268 ff.). Hinsichtlich der E-Portfolio-Forschung wird abschließend für einen design- bzw. gestaltungsorientierten Zugang plädiert (ebd., 260 ff.; siehe weiterführend Kerres, 2012; Reinmann \& Sesink, in Druck).

\section{Fazit und weiterführende Überlegungen}

Die vorliegende Dissertationsschrift leistet einen beachtenswerten Beitrag im gegenwärtigen Diskurs um den Einsatz von E-Portfolios an der Hochschule und ist somit für Medien- und Hochschuldidaktiker sowie Medienpädagogen und Hochschulforscher gleichermaßen interessant: Durch den erheblichen konzeptionellen Anteil gelingt es dem Verfasser, die mit dem qualitativen Forschungszugang einhergehenden Notwendigkeiten hinsichtlich Transparenz der Darstellung und Reflexion seiner Rolle einzuhalten. Die empirischen Befunde sind überzeugend und einfach zugleich: So deutet sowohl das oben skizzierte 4-Phasen-Modell als auch die 3-Schichten-Struktur an, dass der E-Portfolio-Einsatz von wenigen persönlichen, strukturellen und technischen Einflussgrößen abhängig ist, die sich in der praktischen Umset- 
zung in der Hochschule als Grundbedingungen erweisen. Auch wird das vielfach angenommene Spannungsfeld der E-Portfolio-Nutzung zwischen der Person und ihrer sozialen Umwelt empirisch nachgewiesen. Positiv ins Gewicht fällt zudem die persönliche Note, die die Arbeit durchweg auszeichnet. Die theoretischen Rückschlüsse hinsichtlich der Reflexion als bedeutendes Moment der E-Portfolio-Arbeit wirken allerdings mitunter künstlich, da die Anleihen (unter anderem bei Dewey) von Beginn an «auf der Hand» liegen und hier vor allem die Grounded Theory ihre Grenzen erkennen lässt. Auch bietet die Einzelfallstudie zwar wertvolle Einblicke in den studienbegleitenden Einsatz von E-Portfolios im Studiengang eEducation und unterstützt dessen Re-Konzeption. Die Gültigkeit der Ergebnisse über den Fall hinaus löst aber durch die Wahl der Begriffe (Strategie, Modell) beim Lesenden möglicherweise Irritationen aus: Während manche Befunde aufgrund des fallorientierten Zugangs eine eingeschränkte Gültigkeit besitzen, werden andere sehr differenziert dargelegt, sodass vielleicht nicht die direkte Übertragung, wohl aber ähnliche Untersuchungen der E-Portfolio-Arbeit an anderen Hochschulen denkbar sind. Insofern bietet die vorgelegte Arbeit vor allem Orientierung für die Implementierung, Begleitung, Evaluation und ReAnalyse einzelfallbezogenen E-Portfolio-Einsatzes. Auch ergibt sich weiterer Forschungsbedarf, der sich aufgrund der konzeptionellen und empirischen Leistung des Verfassers auf andere Forschungszugänge als den qualitativen Zugang fokussieren kann (nicht muss). Thematisch-inhaltlich könnte beispielweise das Spannungsfeld zwischen persönlicher Kompetenzentwicklung und Curriculumentwicklung deutlicher als bislang untersucht werden eine Entwicklungsperspektive für die E-Portfolio-Forschung an Hochschulen, die sich zusätzlich zur hier vorliegenden mediendidaktischen Betrachtungsweise des E-Portfolio-Einsatzes auch von Ideen zu einer pädagogischen Hochschulentwicklung mit Medien leiten ließe.

Sandra Hofhues 


\section{Rezensionen}

\section{Literatur}

Egloffstein, Marc; Baierlein, Jan \& Frötschl, Clemens. ePortfolios zwischen Reflexion und Assessment - Erfahrungen aus der Lehrpersonenbildung. In: MedienPädagogik. 18/2010, S. 2-20.

HimpsI-Gutermann, Klaus. E-Portfolios in der universitären Weiterbildung. Studierende im Spannungsfeld von Reflexivem Lernen und Digital Career Identity. Boizenburg: vwh 2010.

Kerres, Michael. Mediendidaktik. Konzeption und Entwicklung mediengestützter Lernangebote. 3., vollständig überarbeitete Auflage. München: Oldenbourg, 2012.

Meyer, Torsten; Mayrberger, Kerstin; Münte-Goussar, Stephan \& Schwalbe, Christina. Kontrolle und Selbstkontrolle. Zur Ambivalenz von E-Portfolios in Bildungsprozessen. Wiesbaden: VS Verlag für Sozialwissenschaften, 2011.

Reinmann, Gabi \& Sesink, Werner. Entwicklungsorientierte Bildungsforschung. (In Druck), Preprint online verfügbar unter: http://gabireinmann.de/wp-content/uploads/2011/11/ Sesink-Reinmann_Entwicklungsforschung_v05_20_11_2011.pdf [letzter Zugriff: 20.09.2012] 